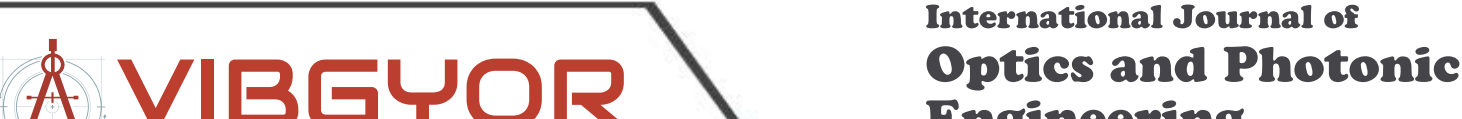 Engineering
}

\section{Oxygen Saturation of Dorsiflexor Muscles during Sustained Isometric Contraction}

\section{Adkham Paiziev* and Fikrat Kerimov}

\author{
Uzbekistan State Institute of Physical Culture, Tashkent, Uzbekistan
}

\begin{abstract}
Sustained isometric contractions of skeletal muscles produce intramuscular pressures that lead to blood flow restriction. Thus, we have the paradox of rising $\mathrm{O}_{2}$ demand due to muscle activity and at the same time reduced blood flow. Aim: To assess muscle oxygenation during sustained isometric low $(30 \%)$, moderate $(60 \%)$ and submaximal (90\% of maximal voluntary contraction (MVC)) contraction of the dorsiflexor muscle. Experiments were conducted on the dominant (right) leg of 8 male students of Uzbekistan State Institute of Physical Culture (age: $19 \pm 2$ years, weight: $75 \pm$ $6 \mathrm{~kg})$. Tissue oxygen saturation $\left(\mathrm{StO}_{2}\right)$ was recorded from the tibialis anterior using near-infrared spectroscopy. $\mathrm{StO}_{2}$ was higher at $30 \%$ compared to both $60 \%$ and $90 \% \mathrm{MVC}$ at all time points after the start of the exercise and higher at $60 \%$ than $90 \%$. This indicates that the supply of $\mathrm{O}_{2}$ did not keep up with its consumption. During an arterial occlusion the minimal $\mathrm{StO}_{2}$ reached $24 \pm 1.77 \%$, which is significantly higher than $\mathrm{StO}_{2}$ during $60 \%$ and $90 \%$ MVC. After each contraction there was a large and immediate hyperaemic response, whose resaturation rate continuously increased from $30 \%$ to $60 \%$ to $90 \%$ MVC. The $\mathrm{StO}_{2}$ resaturation rate was positively correlated with the MVC, indicating a vasodilation depending on the intensity of the exercise.
\end{abstract}

\section{Introduction}

Sustained isometric contraction (SIC) of skeletal muscles produces intramuscular pressure that restricts muscle blood flow $(\mathrm{MBF})$ and limits $\mathrm{O}_{2}$ delivery to tissue [1]. MBF plays a key role in regulating the intensity and type of muscle contractions [2]. A limited MBF due to SIC leads to fatigue due lack of $\mathrm{O}_{2}$ and nutrients. Thus, we have the paradox of rising $\mathrm{O}_{2}$ demand due to muscle activity and at the same time reduced MBF. To clarify this issue much research has been performed mainly on MBF [3-6]. But during exercise there are few measurements of MBF by Doppler ultrasound. Although MBF could be measured by fMRI and PET, this is not possible during training [7].

Previous studies indicate that complete occlusion of MBF occurs at $50-60 \%$ of maximal voluntary contractions (MVC) during SIC $[3,4,6]$. MBF was not occluded at the level of the conduit artery during any of the contraction intensities [3]. Some studies report insensitivity of MBF to the muscle contraction intensity. Thus, our understanding of the oxygenation of the skeletal muscle in response to different intensities of SIC (low, moderate, submaximal MVC) still remains limited.

Despite advantages of fMRI, PET and Doppler ultrasound only one paper has been partly devoted to measure the hemodynamic response of muscles to SIC [3] by near infrared spectroscopy (NIRS). In the near-infrared spectrum $(700-900 \mathrm{~nm})$ light penetrates deeply into the tissue and oxyhemoglobin and deoxyhemoglobin are the strongest absorbers, while myoglobin $(\mathrm{Mb})$ absorbs less. NIRS is an established optical technique to monitor concentration changes of oxyhemoglobin, deoxyhemoglobin, total hemoglobin and tissue oxygen saturation $\left(\mathrm{StO}_{2}\right)$ in a variety of tissues [8,9]. NIRS instruments are non-invasive, small, and applicable in exercise physiology studies.

${ }^{*}$ Corresponding author: Adkham Paiziev, Uzbekistan State Institute of Physical Culture, Tashkent, Uzbekistan, E-mail: adkhampaiziev@gmail.com

Accepted: August 11, 2018: Published: August 13, 2018

Copyright: (c) 2018 Paiziev A, et al. This is an open-access article distributed under the terms of the Creative Commons Attribution License, which permits unrestricted use, distribution, and reproduction in any medium, provided the original author and source are credited. 
The aim was to assess changes in muscle oxygenation during of low, moderate and submaximal SIC of the dorsiflexor muscle.

\section{Methods}

The dorsiflexor muscle was selected, because both venous outflow and arterial inflow can be occluded by a proximal cuff. Without blood supply, the muscle metabolism depends on the $\mathrm{O}_{2}$ in capillaries and muscle cells. Consequently, the oxyhemoglobin and $\mathrm{StO}_{2}$ decrease, while deoxyhemoglobin increases and total hemoglobin remains constant. After the occlusion a hyperemic response occurs, i.e. a rapid increase in oxyhemoglobin, total hemoglobin and $\mathrm{StO}_{2}$, while deoxyhemoglobin is washed-out. From this procedure, we calculated $\mathrm{O}_{2}$ consumption, reoxygenation rate and the half-recovery times of the signals [10].

The recovery baseline (RB) value is the $\mathrm{StO}_{2}$ value after stabilisation during the rest period following a test



Figure 1: Protocol of experiment (top) and example of $\mathrm{StO}_{2}$ trace (bottom). During moderate and submaximal SIC, subjects were not able to perform the exercise for one whole minute. period. The performance baseline is the minimum $\mathrm{StO}_{2}$ value reached during SIC.

Student's t-test was used to compare $\mathrm{StO}_{2}$, desaturation rate and resaturation rate at slow, moderate and submaximal MVC. All data were analyzed using the statistical software package "Statistica" for Windows (version 13). Statistical significance was set at 0.05 .

\section{Results}

Figure 1 displays a typical measurement in one subject. $\mathrm{PB}$ decreased from low to moderate to submaximal SIC30\% MVC (Table 1). Figure 2 compares the $\mathrm{StO}_{2}$ traces for different intensities of SIC. $\mathrm{StO}_{2}$ was higher at low compared with both moderate and submaximal MVC $(\mathrm{P}<0.05)$ at all time points after the start of the exercise and higher at moderate than submaximal MVC ( $\mathrm{p}$ $<0.05)$. Desaturation rate (De, Table 1) increased from slow $(30 \%)$ to moderate $(60 \%)$ to submaximal contractions $(\mathrm{p}<0.05)$. Trends of De as a function of MVC are shown in Figure 2. After each contraction there was a large and immediate hyperemic response (Figure 1). The resaturation rate (Re) of $\mathrm{StO}_{2}$ after SIC depends on the intensity of the SIC and reflects the integrity and functionality of vascular system. It corresponds to a blood vessel vasodilation in response to the SIC. Re increased from slow to moderate $(\mathrm{p}<0.05)$ but remained similar for moderate to submaximal SIC (Table 1 and Figure 1).

During the arterial occlusion the minimal $\mathrm{StO}_{2}$ was 52\% (Table 1 and Figure 1), which is significantly higher than $\mathrm{StO}_{2}$ after moderate or submaximal SIC.

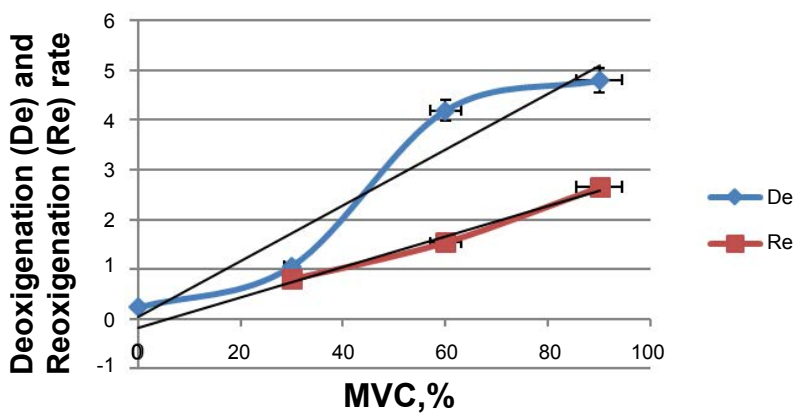

Figure 2: Deoxygenation and reoxygenation rate depend on the intensity of the sustained isometric contraction. MVC $=$ Percent of maximal voluntary contraction.

Table 1: Muscle $\mathrm{StO}_{2}$ parameters during sustained isometric contractions and arterial occlusion $(\mathrm{AO})$. $\mathrm{F}=\mathrm{Force}$; $\mathrm{RB}=\mathrm{Baseline}$ during rest; $\mathrm{PB}=$ Performance baseline; $\mathrm{De}=$ Desaturation rate during contraction and $\mathrm{AO} ; \mathrm{Re}=\mathrm{Resaturation}$ rate after contraction and $\mathrm{AO} ; \Delta \mathrm{StO}_{2}=$ Difference between rest and minimum during contraction and $A O$. Values in mean $\pm \mathrm{SD}$.

\begin{tabular}{|c|c|c|c|c|}
\hline MVC & $30 \%$ & $60 \%$ & $90 \%$ & AO \\
\hline $\mathrm{F}(\mathrm{N})$ & $5.40 \pm 1.03$ & $10.90 \pm 1.03$ & $16.40 \pm 1.03$ & - \\
\hline RB (\%) & $73.16 \pm 0.29$ & $73.08 \pm 0.87$ & $78.66 \pm 3.17$ & $77.75 \pm 3.46$ \\
\hline PB (\%) & $55.33 \pm 0.30$ & $21.58 \pm 0.75$ & $18.15 \pm 3.40$ & $51.35 \pm 2.24$ \\
\hline De $(\% / s)$ & $-1.06 \pm 0.09$ & $-4.19 \pm 0.16$ & $-4.80 \pm 0.16$ & $-0.23 \pm 0.03$ \\
\hline $\operatorname{Re}(\% / \mathrm{s})$ & $0.84 \pm 0.19$ & $1.54 \pm 0.25$ & $2.65 \pm 1.44$ & $2.5 \pm 0.08$ \\
\hline$\Delta \mathrm{StO}_{2}(\%)$ & $16.83 \pm 4.62$ & $54.5 \pm 9.24$ & $59.51 \pm 5.14$ & $26.45 \pm 1.77$ \\
\hline
\end{tabular}




\section{Conclusion}

- The sharp decreases in $\mathrm{StO}_{2}$ after the start of moderate and submaximal SIC, indicates that the blood vessels are occluded due to intramuscular pressure. This indeed shows that $\mathrm{O}_{2}$-delivery is impeded and cannot cope with the increased $\mathrm{O}_{2}$-consumption.

- $\mathrm{StO}_{2}$ resaturation rate $(\mathrm{Re})$ permanently increased from low to moderate to submaximal contractions. This reflects the resaturation of haemoglobin, which depends on integrity and functionality of vascular system and reflects blood vessel vasodilation.

\section{Acknowledgement}

The authors express their gratitude to the Swiss National Science Foundation to support this work via grant no. IZ74Z0_137423 (S-84301-05-01).

\section{References}

1. Degens H, Salmons S, Jarvis JC (1998) Intramuscular pressure, force and blood flow in rabbit tibialis anterior muscles during single and repetitive contractions. Eur J Appl Physiol Occup Physiol 78: 13-19.

2. Saltin B, Radegran G, Koskolou MD, Roach RC (1998) Skeletal muscle blood flow in humans and its regulation during exercise. Acta Physiol Scand 162: 421-436.

3. Chris J McNeil, Matti D Allen, Eric Olympico, J Kevin Shoe- maker, Charles L Rice (2015) Blood flow and muscle oxygenation during low, moderate, and maximal sustained isometric contractions. Am J Physiol Regul Integr Comp Physiol 309: 475-481.

4. Sjogaard G, Savard G, Juel C (1988) Muscle blood flow during isometric activity and its relation to muscle fatigue. Eur J Appl Physiol Occup Physiol 57: 327-335.

5. Vollestad NK, Wesche J, Sejersted OM (1990) Gradual increase in leg oxygen uptake during repeated submaximal contractions in humans. J Appl Physiol 68: 1150-1156.

6. Wesche $\mathrm{J}$ (1986) The time course and magnitude of blood flow changes in the human quadriceps muscles following isometric contraction. J Physiol 377: 445-462.

7. Hughson RL, Shoemaker JK, Tschakovsky ME, Kowalchuk JM (1996) Dependence of muscle VO2 on blood flow dynamics at onset of forearm exercise. J Appl Physiol 81: 1619-1626.

8. M Wolf, M Ferrari, V Quaresima (2007) Progress of near-infrared spectroscopy and topography for brain and muscle clinical applications. J Biomed Opt 12: 062104.

9. T Hamaoka, KK McCully, V Quaresima, K Yamamoto, B Chance (2007) Near-infrared spectroscopy/imaging for monitoring muscle oxygenation and oxidative metabolism in healthy and diseased humans. J Biomed Opt 12: 062105.

10. Vasiliki Gerovasili, Stavros Dimopoulos, Georgios Tzanis, Maria Anastasiou-Nana, Serafeim Nanas (2010) Utilizing the vascular occlusion technique with NIRS technology. International Journal of Industrial Ergonomics 40: 218-222. 